\author{
Bartosz Wójcik \\ ORCID: 0000-0002-8618-057X \\ Universität Szczecin, Szczecin
}

DOI: $10.19195 / 0435-5865.143 .3$

\title{
Pommersche Identitäten. Zu Der Teufel vom Sande Hans Hoffmanns
}

\begin{abstract}
s
Der 1891 von Hans Hoffmann veröffentlichte Text Der Teufel vom Sande erschien in einer Novellensammlung, die sich mit der Geschichte und dem geläufigen Bild Pommerns auseinandersetzte. Der damals populäre Text schildert eine fiktive Geschichte vor historischem Hintergrund. Neben seiner Unterhaltungsfunktion - die Liebesgeschichte ist für den Plot relativ wichtig - spielt die literarische Auseinandersetzung mit dem Kulturerbe und der Identität der Pommern um 1900 eine gewichtige Rolle. Auf Stereotypen basierend - und diese humoristisch nutzend - bot Hoffmann eine Vision Pommerns an, die zum Zeitpunkt der Entstehung des Deutschen Reiches von Bedeutung war. Heute kann sie nur noch historisch rekonstruiert werden.
\end{abstract}

Schlüsselwörter: Pommern um 1900, Hans Hoffmann, Regionalliteratur

\section{Pomerania's identities. On Hans Hoffmann's Der Teufel vom Sande}

Der Teufel vom Sande was published in 1891 in a collection of short stories which tackled the history and the common image of Pomerania. The text, classified as historical fiction, was popular at the time of its publishing. Apart from its role as a piece of entertainment - a love story plays a relatively important part in the narrative - the short story divagates about the cultural heritage and the identity of Pomerania's inhabitants around 1900. Basing on stereotypes - and using them for humorous purposes - Hoffmann proposed a vision of Pomerania that was of vital significance at the time of the German Reich's foundation. Nowadays it can only be historically reconstructed.

Keywords: Pomerania around 1900, Hans Hoffmann, regional literature

Bartosz Wójcik, Uniwersytet Szczeciński, Instytut Filologii Germańskiej, Al. Piastów 40b, bud. 5, 71-065 Szczecin, Polen, E-Mail: bartosz.wojcik@usz.edu.pl

Received: 29.09.2017, accepted: 4.05.2018

Germanica Wratislaviensia 143, 2018

(C) for this edition by CNS 
I.

Regionalbezogene Themen sind in der in Pommern geschriebenen Literatur seit Langem bekannt. Sie bildeten eine Mischung aus Beiträgen zur regionalen Kulturgeschichte mit mythenartigen Schilderungen voll von wahren und fiktiven Gestalten sowie historisch bedeutsamen Orten, allgemeinen Weisheiten zur menschlichen Natur und purer Unterhaltung. Nicht aus den Augen gelassen werden sollte dabei die große Idee hinter dem Erzählstoff. Denn all of the great novels, however, and most of the good ones, are not just telling a story but are pursuing an idea. They are about something significant, and this theme is just as important as the story line, if not more so, wie Linda Seger es formuliert (Seger 1992: 14).

Das geschichtliche Geschehen scheint den Hintergrund auszuschmücken und zugleich der eigentliche Urheber für die Textentstehung (,something significant") zu sein; gleichzeitig konstituiert jedoch jenes Geschehen die Gegenwart der Bewohner eines bestimmten Gebiets. Mit anderen Worten, die Identität der Menschen als der alltägliche Bezug zum Hic et Nunc, den der Leser realisiert - wird auf der Basis der Geschichte fundiert und mit dem Instrument der Literatur (re)konstruiert. Denn „Geschichte zu schreiben, heißt, das Gedächtnis wiederzufinden“, und „eine Gesellschaft kann genauso wenig wie ein Individuum wahrhaftig ohne Erinnerung leben“, wie Jan M. Piskorski es im Schlusswort zum ersten deutsch-polnischen Sammelband zur Geschichte Pommerns zum Ausdruck bringt (Piskorski 1999: 423 f.). Im ausgehenden 19. Jahrhundert waren es historische Transformationsprozesse, der rasante technologische Wandel sowie wissensgeschichtliche Faktoren, ${ }^{1}$ die auch heute für die enorme Entwicklung der Gedächtnisforschung sorgen - damals waren sie allerdings weniger ausgeprägt als in den nachkommenden Dezennien -, die die Konjunktur der Erinnerungskultur ankurbelten. Ähnlich funktionierte auch die fundamentale Medialität der Erinnerung verstanden als Austausch von Inhalten, die anderen mitteilbar sind und die dafür ein Medium (hier: Sprache) benötigen. Die Rolle des Mediums spielte um die Jahrhundertwende in großem Maße die Heimatliteratur, wo fiktive Gestalten vor wahrem historischem Hintergrund agierten. ${ }^{2}$ Diese Art von Literatur war in Pommern - ähnlich wie in anderen Regionen Deutschlands - im II. Deutschen Reich populär. Unter vielen anderen bearbeitete Ernst Gollnow das Thema der Einführung des Christentums in Pommern (Gollnow 1893, 1901). Über den pommerschen Herzog Svantepolk II. schrieb Hans Werder (Werder 1901), über den Siebenjährigen Krieg Franz Adam Beyerlein (Beyerlein 1906). Neben Romanen entstanden zahlreiche Erzählungen, Sagen, Gedichte und Dramen, die sich mit der Geschichte der Region auseinandersetzten und diese vergegenwärtigten.

${ }^{1}$ Ausführlicher zu diesen Kategorien in Bezug auf das 20. Jahrhundert s. Erll (2005); Rosenfeld (2009: 122-158).

${ }^{2}$ Gemeint ist in erster Linie die Heimatliteratur in der Tradition Walter Scotts. Hierzu vgl. u.a. Limlei (1988); Borgmeier / Reitz (1984). 
In den meisten Texten, die die regionale Geschichte zum Thema haben, lassen sich - neben angestrebtem Amüsement der Leserschaft - Allusionen zur unterhaltenden Identitätsstiftung auf der Basis historischer Inhalte feststellen, die einen dem Autor zeitgenössischen Bezug herzustellen scheinen. So etwa glaubt Horst Hartmann im Drama Witzlaw von Rügen Kruses von 1881 eine Parallele zu seiner (d.i. Kruses) Zeit zu finden. Im Werk, dessen Handlung im 14. Jahrhundert spielt, ist nach Hartmann eine dualistische Wiederholung der Geschichte durch deren literarische Bearbeitung festzustellen: „Was hier als Beginn des Übergangs von der Feudalordnung zur bürgerlichen Ordnung als Hauptbotschaft gestaltet worden ist, signalisiert um 1880 das Ende der »Gründerzeit« und die Ankündigung des Übergangs zum Imperialismus" (Hartmann 2010: 70). Dass der zeitgenössische Bezug als ein wichtiges Kriterium für die Entscheidung über die Publikation des jeweiligen Textes galt, belegen die Schwierigkeiten Wilhelm Jensens, seine Novellensammlung Aus schwerer Vergangenheit zu drucken: „Es wollte allerdings überhaupt keine [Zeitschrift - Anm. BW] den gesamten Novellencyclus, sondern jegliche nur einzelne derselben veröffentlichen; die von ihrem Publicum erheischte »Actualität« fehle ihnen zu sehr, um ein derartiges Wagniß mit fünf Erzählungen zu unternehmen“" (Jensen 1901: VII). ${ }^{3}$

Im Folgenden soll die Rolle der Regionalgeschichte als literarischer Träger identitätsstiftender Inhalte im Hinblick auf die Gegenwart des Autors in der Novelle Der Teufel vom Sande des Schriftstellers Hans Hoffmann exemplarisch untersucht werden.

\section{II.}

Hans Hoffmann, im Jahre 1848 geborener Stettiner, war Sohn eines Predigers und relativ früh Schüler des bekannten regionalen Dichters Heinrich Ludwig Theodor Giesebrecht. ${ }^{4}$ Bereits während seiner Studienzeit weilte er in verschiedenen Regionen Deutschlands - in Berlin, Halle und Bonn. Nach seiner Promotion unterrichtete er als Lehrer an Gymnasien in Stettin (heute: Szczecin), Stolp (heute: Słupsk), Danzig (heute: Gdańsk) und Berlin. Er unternahm Reisen nach Italien, Griechenland und in den Norden, lebte auch eine Zeitlang in Rom, wo er als Hauslehrer arbeitete. Allmählich wuchs seine Bedeutung für das deutsche Schrifttum: In den Jahren 1884-1886 redigierte er die Deutsche Illustrierte Zeitung, und wenige Jahre später, 1902, wurde er zum Generalsekretär der Schiller-Gedächtnis-Stiftung in Weimar.

Das Schaffen des bis heute gelesenen Autors (vgl. Neuauflagen, u.a. Hoffmann 2013, Hoffmann 2014) wurde von seinen Zeitgenossen hoch geschätzt. Edu-

${ }^{3}$ Allerdings waren diese Erzählungen recht umfangreich: Sie umfassten insgesamt 355 Seiten in zwei Bänden.

${ }^{4}$ Die Informationen zum Lebenslauf Hans Hoffmanns stützen sich auf Raeck (1972: 335). 
ard Engel lobte in seiner Geschichte der Deutschen Literatur des Neunzehnten Jahrhunderts und der Gegenwart seine „Begabung für das Ersinnen und Entfalten einer guten Geschichte“ (Engel 1908: 285) und nannte ihn neben solchen Größen wie Fontane, Stifter oder Storm, deren Kenntnis ,zur Berechtigung der Lehrer für die oberen Klassen verlangt [wird]" (Engel 1908: 463). 1907 würdigte das Meyers Konversations-Lexikon Hoffmann folgendermaßen: „H. gehört zu den besten Lyrikern und Novellisten der Gegenwart, er ist einer der wenigen, die die edle Tradition Storms und Kellers fortführen. Große Anmut der Form und Sprache, inniges Naturgefühl und tiefer Humor zeichnen seine Erzählungen aus“" (Lexikon 1907: 420). Noch zu Lebzeiten des Schriftstellers erschienen einige Studien über sein Werk (z.B. Ladendorf 1908; Arminius 1909). Seine spätere Einschätzung fällt weniger positiv aus: Johannes Mumbauer äußerte in den 1930er Jahren seine Meinung über ein ,überaus fruchtbares, aber von seinen Verehrern überschätztes Talent [...]; Hoffmann, der die Art der älteren »poetischen Realisten « Keller, Meyer, Storm, Reuter und Jensen nicht ungeschickt verjüngte, aber auch verwässerte und ins philisterhaft »Gemütliche« abdämpfte“ (Mumbauer 2015: 68) blieb für ihn ein Autor, der die Bedürfnisse seiner zeitgenössischen Leserschaft ahnte, dessen Werk jedoch im Laufe der Zeit an autonomer universeller Kraft verlor. Denselben Aspekt unterstrich Christian Schwarz, gerade im Kontext seiner historischen Romane: „Die histor. Romane versuchen, wenig erfolgreich, historisch-polit. Aspekte mit persönl. Schicksalen der handelnden Figuren zu verbinden, u. führen in die Nähe der Heimatkunst." (Schwarz: Hans Hoffmann, in: Kühlmann 2009: 525). Auf der anderen Seite nannte Roswitha Wisniewski, Autorin des wichtigsten Buches zur Geschichte der Regionalliteratur Pommerns, Hans Hoffmann neben Theodor Fontane und Anna Bonin als Autoren, ,die, namentlich auf Pommern bezogen, Lebensstil, gesellschaftliche Ordnung und Wertvorstellungen jener Zeit in ihrer Geltung und in ihrer Fragwürdigkeit gezeichnet haben" (Wisniewski 2013: 246), und bezeichnete seine Belletristik als ,eine ironische, fast parodistische und dennoch liebevolle Vorführung dessen, was preußische Lebenshaltung nach dem damaligen Verständnis ausmachte“ (Wisniewski 2013: 248).

Besonders die letztgenannten Züge lassen sich in der Novelle Der Teufel vom Sande finden. Sie ist die längste der insgesamt vier, die Hoffmann in den $G e-$ schichten aus Hinterpommern zusammenstellte. ${ }^{5}$ In der auktorialen Erzählsituation wird zunächst einmal die legendäre Entstehung einer ersten Sanddüne an der Ostseeküste in der Umgebung von Köslin (heute: Koszalin) geschildert. Das erzählerische Interesse verschiebt sich jedoch schnell auf das 17. Jahrhundert, ${ }^{6}$ und auf die Familie von Kieköwer mit Jochen vel Joachim als Herrn, die ein großes Gebiet in dieser Gegend innehatte. Ins Haus wird ein Waisenkind als Knecht

${ }^{5}$ Erstausgabe 1891, im Folgenden zitiert nach der Ausgabe Hoffmann 1905. Andere drei Novellen sind: Der grobe Pommer, Der Tribuliersoldat und Der falsche Bogislaw.

${ }^{6}$ Erwähnt wird der letzte pommersche Herzog Bogislaw XIV., der in den Jahren 1625-1630 regierte. 
geholt, der die beiden Töchter der Herrschaften, Kathinka und Valeska, betreut. Für ihren tagtäglich getriebenen Unfug wird er regelmäßig öffentlich verprügelt. Seine schreckliche Vergangenheit wird dem Leser in Details unbekannt bleiben. ${ }^{7}$ Seine Freizeit im Alltag verbringt er im zunehmenden Maße mit dem Pastor, der ihm viel praktisches Wissen, Lektüre wie philosophische und religiöse Denkanstöße beibringt. Schlussendlich, um für ein Vergehen der Mädchen nicht stellvertretend bestraft und ,unbetrauert von Kathinka [gehängt - Anm. BW] in den öden Lüften herumbaumeln zu sollen“ (Hoffmann 1905: 176), flieht er.

Inzwischen entsteht eine kleine Gemeinde auf einem in der Einöde liegenden Sandstreifen. Eine winzige Vertretergruppe übergibt das Dorf dem Pommernherzog Bogislaw XIV. in Stettin, ohne eine weitere Lehnsabhängigkeit. Nach ein paar Jahren erscheint dort Barnim. Zunächst wird er zum Priester und anschließend zum Anführer der Gemeinschaft gewählt. Der durch das allnächtliche Ringen mit dem Satan in seinem Wagen bekannte Geistliche setzt sich aktiv für das Dorf ein. In seiner Freizeit treibt er den Kirchenbau sowie die Anlage der Strauchzäune um das Dorf zum Schutz gegen den Sand und Menschen voran.

Da inzwischen der Herzog verstorben ist und sich das Land in den Händen Brandenburgs befindet, ${ }^{8}$ kommt ein Abgesandter des Kurfürsten, Herr von Luchtemar, an. Da Barnim weder die Obrigkeit Brandenburgs akzeptiert noch einem Posten im brandenburgischen Dienste zustimmt, wird er in Absprache mit Luchtemar von den beiden Kieköwer-Töchtern - von denen er sich wieder hat erkennen lassen - entführt. Briefe von Valeska, die vor der Abreise von Luchtemars zu seiner Frau geworden ist, berichten inzwischen von einer Schlacht der Dorfbewohner gegen die Polen, die zwar die Gemeinde letztendlich nicht belagert, aber einen großen Waldteil verwüstet haben. Ein dadurch geförderter Sandsturm vernichtet anschließend das Dorf; Joachim von Kieköwer stirbt vor Kummer. Die Gemeinde bekommt etwas Land von der neuen Herrin, Kathinka. Der aus Berlin zurückgekommene Barnim wird von ihr mit Liebe empfangen, und beide heiraten.

Bereits die einführenden Worte zu Der Teufel vom Sande unterstreichen die hinterwäldlerische Insellage Pommerns: Im ersten Satz wird vorweggenommen, die zu schildernde Geschichte wird sich ,ganz hinten im allerhintersten Hinterpommern“ (Hoffmann 1905: 147) abspielen. Damit wird auf die Wahrnehmung der Provinz als einer von der Zivilisation abgelegenen Gegend hingedeutet. Die wüstenanmutende, „fremdartige und schier grausige Schönheit der wilden Dünenberge“" (Hoffmann 1905: 147) bei Leba (polnisch: Łeba, nordöstlich von Stolp) bildet die Kulisse, die an einigen Stellen in den Vordergrund treten. Der Text würdigt sowohl eine außer-

7 „Alle anderen Erinnerungen waren in den Schrecknissen der jüngsten Zeit als in einem blutigen Meere untergegangen“" (Hoffmann 1905: 160).

${ }^{8}$ Während des Dreißigjährigen Krieges wurde Pommern von Schweden überfallen und zum Teil von Brandenburg belagert. Es folgte eine Zeitspanne der Konflikte zwischen Schweden, Brandenburg und zum Teil Polen, die im pommerschen Gebiet ausgetragen wurden. Erst nach dem Wiener Kongress 1815 wurden alle Teile Pommerns in preußischer Hand als eine Provinz vereinigt. 
gewöhnliche pommersche Naturerscheinung als auch die Entstehung und Wechselwirkung mit dem Leben der Pommern - in der Geschichte, die in die Gegenwart des Schriftstellers hineinspielt - auf mythologisierende Weise zu erklären. Sowohl die Genesis der Landschaft als auch die Geschichte der in dieser unbewohnbaren Gegend lebenden Gemeinschaft trägt viel Neues zum Verständnis der Region und der dortigen Menschen bei. Beide Aspekte - der natürliche und der der Geschichte der Menschen - werden symbolisch im Naturbild vereinigt: einer kargen, aber außergewöhnlichen Landschaft, mit der sich nur hartgesottene Menschen abfinden können. Auf diese Weise trägt das Pommern Hoffmanns viele aus anderen regionalen Texten bekannte Klischees, hebt sie jedoch zugleich zu einer symbolischen Figur empor. Dieses Konstrukt wird zugespitzt, indem Assoziationen ungezähmter, naturgewaltiger und menschenunfreundlicher Wildnis genannt werden: „Denn es sei kein Zweifel, in diesem wilden Boden könne nicht einmal ein Gänseblümchen haften, geschweige denn anständige Rosen oder Lilien. Ganz ebenso gut könne man in der afrikanischen Wüste oder auf einem grönländischen Gletscher einen Ziergarten anlegen" (Hoffmann 1905: 152). Pommern als fast unberührtes Land - in der Natur wie im übertragenen Sinne, als intellektuell öde Kulturlandschaft ${ }^{9}$ - ist dementsprechend nicht prädestiniert, „für menschliche Historie einen Schauplatz abzugeben“ (Hoffmann 1905: 177). Darin offenbart sich das Anliegen des Autors, die Vorteile der Region beim Spiel mit dem Klischee zu zeigen. Obwohl die Hoffmannsche Heimat tatsächlich ,zur Kulisse [wird], hinter der sich ganz anderes abspielt“, wie Herrmann Bausinger dies für die historisierende Verwendung des Begriffs erläutert, entspricht die Heimat Hoffmanns jedoch nicht dem von Bausinger gemalten Bild, laut dem sie ,vielfach auf einen - freilich nicht gerade kleinen - Satz von Fertigbauteilen reduziert [wurde], die immer wieder nur schöne Fassaden ergeben. Heimat wurde in und an Stereotypen festgemacht, die in einem vordergründigen Reiz-Reaktion-Verhältnis Behaglichkeit erzeugen. Heimat wurde und wird von der Stange geliefert" (Bausinger 1990: 83).

Der Teufel vom Sande liefert die Heimat jedoch nicht von der Stange. Einerseits bestätigt der Text das ,exotische“ Image der Region, deren geographische Randlage und entlegener Provinzcharakter damals schon als touristisch angelegte Elemente der Marketingstrategie verstanden - also auch affirmativ wahrgenommen - werden konnten: als Gegensatz zur Moderne und dem damit einhergehenden Gefühl von „Utopie und Alptraum“ (vgl. Bolz 1997: Web.). Andererseits liefert er ein Gruppenportrait der Protagonisten, das zu einem Bild vom „Pommer“ schlechthin - eben als Kollektivsingular - mit allen seinen ihm typischen, wenn auch stereotypisch vereinfachten, Alleinstellungsmerkmalen werden sollte. Seine Strategie der Sympathielenkung basiert auf Zusammenstellungen von pejorativ

${ }^{9}$ Die Geläufigkeit dieses Stereotyps bestätigt eine geradezu identische Feststellung in einem Sachbuch - einem Reiseführer - aus jener Zeit: „In vielem was zum feinern Lebensgenuss gehört, wird freilich so mancher, den sein Schicksal hierher [d.h. nach Stettin-Anm. BW] verschlägt, etwas Resignation üben müssen“ (Stettin: 16). 
anmutender Begrifflichkeit mit Bezügen auf pommersche Eigenart. Dies erfolgt in einzelnen Formulierungen wie „die Leutchen aber hatten ihre Schlauheit, obgleich sie Pommern waren" (Hoffmann 1905: 179) bzw. in Anekdoten: Der Gesandte des brandenburgischen Kurfürsten hatte vor, Barnim von Kieköwer zu entführen und ihn in Berlin als ,ein rechtschaffenes hinterpommerisches Kuriosum seinem Kurfürsten vorzustellen“ (Hoffmann 1905: 249 f.). Der von Hoffmann woanders gründlich bearbeitete Stoff ${ }^{10}$ war kein Novum in der Literatur Pommerns. Der Pädagoge, Historiker und Lexikograph Christian Schöttgen veröffentlichte 1721 die Anekdote (Schöttgen 1721), die er bei Erasmus Francisci (Francisci 1663) gefunden haben wollte. Das damals gängige stereotype Bild von Pommern ${ }^{11}$ übt hier jedoch womöglich eine zusätzliche Funktion zur unterhaltenden Identitätsstiftung aus. Es stellt sich die Frage, ob Hoffmann für seine Erzähleffekte auch Seitenblicke auf die zeitgenössische Anthropologie nutzte? Angehörige einzelner Ethnien wurden bekanntlich als Exoten auf sog. Kolonialausstellungen bzw. Völkerschauen gerade nach der Reichsgründung und bis in die 1930er Jahre hinein öffentlich zur Schau gestellt. Es lässt sich nicht eindeutig die Frage beantworten, ob Hoffmann lediglich eine alte Anekdote verarbeitete oder doch eine solche auch in Zeitbildern spiegelte - jedoch liegt diese Vermutung nahe.

Die Möglichkeit für diese Strategie verleihen ihm die gewählten Erzählmodi. Die Handlung wird sowohl narrativ als auch durch Dialoge getragen. Die Figuren sprechen, wobei sie mit zusätzlichen Informationen zu ihren emotionalen Zuständen - gerade mit dieser wechselnden Erzählperspektive - versehen werden. Die Distanz, die durch die Vermittlung des Erzählers zustande kommt, wird in einigen wenigen Fällen durch den Umstand gemildert, dass dieser nicht in allen Situationen allwissend ist. In Formulierungen wie: „Es ist unbekannt, welche Erinnerung ihm die Kenntnis zutrug, daß freilich das Prügeln zu den altväterlichen Gerechtsamen einer ehrlichen pommerischen Ehe gehört" (Hoffmann 1905: 164, Unterstreichung: BW) offenbart sich die variierende Erzählperspektive, die zwischen der auktorialen und der personalen schwankt. Auf diese Weise bekommt der Leser ein quasi-objektiviertes Bild, das aus mehreren Blickpunkten beleuchtet wird. Diese lassen den Glaubwürdigkeitsgrad der Beschreibung der dargestellten Welt scheinbar steigern.

III.

Die Aktualität der historischen Thematik wird deutlich, wenn es um die Frage der nationalen Zugehörigkeit der Bewohner der Region geht. Nicht zufälligerweise

10 Vgl. den Inhalt der Novelle Der grobe Pommer im selben Buch Hoffmanns.

11 Vgl. z.B. die Vorstellungen von Effi Briest, der Hauptprotagonistin des gleichnamigen Romans von Theodor Fontane. Bei Überlegungen zum Ort in Pommern, wo sie leben sollte, ,gefiel [sie] sich nämlich darin, Kessin als einen halbsibirischen Ort aufzufassen, wo Eis und Schnee nie recht aufhörten" (Fontane 2010: 22). 
platziert Hoffmann die Handlung in der ersten Hälfte des 17. Jahrhunderts. Sie umfasst mehrere Jahre, ${ }^{12}$ vermutlich von den 1620ern bzw. 1630ern bis in die 1650er, mit dem Höhepunkt um das Jahr 1648, ${ }^{13}$ also einen besonders schwierigen Moment in der pommerschen Geschichte. Fast die gesamte Zeitspanne des Dreißigjährigen Krieges bildet eine dramatische Kulisse der Handlung in einer abgelegenen Provinz Hinterpommerns. ${ }^{14}$ Das Herzogtum Pommern, zwischen dem 12. und 17. Jahrhundert auf der Landkarte Europas präsent, war mehrere Jahrhunderte lang politisch und militärisch zu schwach, um gegenüber den benachbarten größeren Herrschaftshäusern Polen, Brandenburg und dem Heiligen Römischen Reich Deutscher Nation unabhängig zu bleiben. 1637 verstarb Bogislaw XIV. ohne männliche Nachkommen, wodurch das pommersche Herzoghaus des Greifengeschlechts erlosch. So wurden Schweden, Brandenburg und Polen zu Interessenten um die Region. Schlussendlich wurde der Lehnsherr Brandenburg und der Lehnsempfänger Schweden. Pommern wurde in drei Teile geteilt. ${ }^{15}$ Die Entwicklung des nationalen Gedankens im politischen Sinne, der sich zur Identitätsfrage entfaltet, bildet große, wenn auch in der Novelle zerstreute und häufig in der Handlung latent fungierende Textteile.

Als Feststellung nach der Teilung Pommerns in einem schwedischen, brandenburgischen und polnischen Teil meint der Anführer der Gemeinde „Sand“, Priester Barnim: „Von jedem pommerischen Edelmanne zu allermeist aber fordere ich auch mehr, fordere ich recht heilige Ehrfurcht und Ergebenheit: denn dieser schmale Landsaum hier ist jetzt das alleinige Pommern geworden, und ein andres gibt es nicht mehr: ich aber bin der Herzog in diesem Pommern!" (Hoffmann 1905: 227 f.). Die wie ein Coup Barnims anmutende Aussage ${ }^{16}$ ist im Lichte der

${ }^{12}$ Am Anfang sind die beiden Töchter noch nicht geboren, in der Abschlussszene heiratet Kathinka Barnim. Dementsprechend umfasst die Handlung wahrscheinlich circa 25-30 Jahre.

${ }^{13}$ An einer Stelle ist die Rede von einem Friedensvertrag, der ,bald“ in Westfalen ,zusammengebraut" werden soll (Hoffmann 1905: 200). Gemeint ist der sog. Westfälische Frieden, d.h. die in Münster und Osnabrück zwischen Kaiser und Reich, Frankreich und Schweden und deren Verbündeten abgeschlossenen Verträge, die 1648 den Dreißigjährigen Krieg beendeten.

14 Gelegentlich werden kriegsbedingte Situationen erwähnt, wie etwa: „Liebe Verbündete des guten Herzog Bogislaw kamen ins Quartier, die tapferen Wallensteiner, und räumten binnen kurzem [...] fast so alles gründlich auf" (Hoffmann 1905: 156). Sie treten jedoch niemals in den Vordergrund der Handlung.

${ }^{15}$ Das Gebiet um Leba und Lauenburg (heute: Lębork) (mit einem separaten Teil um Bütow, heute: Bytów) wurde zur brandenburgischen Erwerbung 1658 und als solche an Preussisch-Hinterpommern angeschlossen (vgl. Buchholz: 61).

${ }^{16}$ Es darf von der Handlung im Text abgesehen werden, zu dessen Schluss er seiner geliebten Kathinka gestand, er hatte um ein freies Land nicht nur für Pommern kämpfen wollen, sondern um seine soziale Position zu stärken, damit er sie - die Tochter eines Großgrundbesitzers - gewinnen konnte (Hoffmann 1905: 283 f.). Der Liebesplot funktioniert auf der Unterhaltungsebene des Textes, die sicherlich eine gewichtige Rolle für den zeitgenössischen Leser gespielt haben musste und so für die Popularität des Textes sorgte (davon zeugen mehrere Neuauflagen des Buches innerhalb von ca. 30 Jahren); sie ist jedoch von bedingtem Interesse einer historisch angelegten literaturwissenschaftlichen Untersuchung der Novelle. 
Politik und der kulturellen Identität zu betrachten, denn hier offenbaren sich Überlegungen des Verfassers zum Wesen Pommerns im Wandel der Zeiten.

Der Priester betont an einer anderen Stelle, er habe nicht etwa vor, König oder Herzog des staatslos gewordenen Landstreifens zu werden, sondern ein freier Herr vom Sande, „bis wieder ein rechter Herzog im ganzen Pommern regiere“ (Hoffmann 1905: 218 f.). Daraus ergibt sich das Bild eines pommerschen Patrioten, der im Moment einer schwierigen historischen Umwälzung im Dienste seines von Aggressoren zerstückelten Landes die Rolle eines Führers übernimmt, bis das rechtmäßige dynastische Geschlecht die Macht über das Territorium wieder erlangen soll ${ }^{17}$ - oder noch anders formuliert, bis das Land nicht mehr fremden herrschaftlichen Einflüssen untergeordnet ist. Diese sind nicht nur politisch, sondern auch kulturell zu deuten. ${ }^{18}$

Es wird unterstrichen, dass die Pommern sich nicht etwa polnisch fühlten: Eine der Hauptfiguren, Joachim, äußert sich zu dem Problem, dass das Dorf „Sand“ in direkter Nähe von brandenburgisch-polnischen Grenzsteinen liegt, die jedoch vom Sand im Laufe der Zeit verschüttet worden waren. Da dadurch ein potentiell ernstlicher Streitfall entstehen konnte, bekam er Angst, ,auf daß nicht etwa aus eitel Vergessen und Nachlässigkeit ein Fleckchen Landes an den polnischen Nachbar gerathe“" (Hoffmann 1905: 232).

Die Beziehung zum Deutschtum ist zwiespältig. Der Abgesandte des brandenburgischen Kurfürsten ,sah mit Gewissheit, daß die Pommern sich nichts Besseres wünschten, als mit Brandenburg vereinigt zu werden und des Schweden ledig zu sein“ (Hoffmann 1905: 230). Zwar dürfte die Wendung „nichts Besseres“ auch das kleinere Übel angesichts des Verlustes der Unabhängigkeit bedeuten. Diese Auslegung wird durch das Verhalten des Priesters Barnim gekräftigt: Als derselbe Gesandte ihn zur „Anerkennung so edler deutscher Waffenthat“ (Hoffmann 1905: 274) - dem militärischen Vorgehen gegen die Polen - überreden wollte, lehnte dieser seine Argumente ab.

Wenn die Deutschen - verstanden wohl im ethnischen Sinne, wobei die dem Autor zeitgenössische Anspielung auf die nationale Bedeutung des Begriffs vermutet werden kann - erwähnt werden, ist die Wertung positiv. Der Leser erfährt, „nur allein sein Wort zu brechen ist einem deutschen Edelmanne nicht erlaubt, sondern ist Schimpf und Unehre sogar vor seinesgleichen" (Hoffmann 1905: 226). Unklar bleibt jedoch, ob Pommern Deutsche sind oder einfach nur ein pränationaler Stamm. Sicherlich sind sie von den Schwaben, Bayern, Rhein-Bewohnern und Österreichern zu unterscheiden: ,[D]ie möchten Euch vielleicht ein wenig noch fremd bedünken, denn sie sind anders geartet in mancherlei Dingen und im Sprechen nicht immer bequem zu verstehen“ (Hoffmann 1905: 240). Über die

${ }^{17}$ In diesem Lichte erscheint der Name Barnims besonders symbolisch geprägt: So hießen mehrere Herzöge von Pommern.

18 Es kann kein Zufall sein, dass 1871 die bis heute meist zitierte holistische Definition von Kultur für die Kulturanthropologie von Tylor erarbeitet wurde (vgl. Tylor 1873: 1). 
Brandenburger bemerkt ein Pommer: „[W]ir würden mit Freuden zu Euch kommen und zu den Preußen, die auch von gleicher Art und Stamme sind" (Hoffmann 1905: 240). Doch das bedeutet nicht, der preußische Geist würde die Identität mitbestimmen. Der oben erwähnte Brandenburger stellt an anderer Stelle fest: „Ihr bleibet Pommern, auch wenn Ihr Brandenburger werdet" (Hoffmann 1905: 240). Der Pommer antwortet schlussfolgernd: Nicht nur bleibe Pommern geteilt, „also bleibe auch ich so lange getrennt in meinem eigenen Pommern“ (Hoffmann 1905: 241).

So vertritt Hoffmann, wie es scheint, die Meinung, die Pommern seien Einheimische in einem Gebiet, in dem Deutsch gesprochen wird und das dem deutschen Reich angehört. Aber er verfährt diesbezüglich wenig präzise. Möglich ist sogar, dass sich die Pommern als „Pommern deutscher Nation“ bezeichnen würden. Das ,,pommersche“ Element ist dabei unverwechselbar, einfach weil es „pommersch“ ist.

Unabhängig davon, ob die Pommern von einem deutschen, polnischen oder schwedischen Herrn regiert werden, brauchen sie nach Hoffmann keine Amtsperson, die sie kontrollieren müsste. Das abgelegene Hinterpommern sei von lauter guten pommerschen Menschen bevölkert: „Diese Einsamkeit lockte manchen, sich zu bergen, doch keinen, zu rauben“ (Hoffmann 1905: 180). Der Verfasser geht noch weiter und liefert ein idyllisches Bild ohne Richter, Rechtsanwälte und Bildungsverwalter, ja sogar ohne Ärzte:

Des weiteren war ihr Vorteil, daß es dort keinen Richter und keinen Advokaten gab: so blieb ihnen nichts übrig, als sich untereinander zu vertragen, und selbst die Schelme, die unter dem zugefahrenen Volk mit dreinliefen, mussten sich beeilen, ordentlich zu werden. Und keinen Arzt: so waren sie genötigt gesund zu bleiben und starben am Ende in Frieden. Und keinen Schulmeister: so verdarben sich ihre Kinder weder die Augen noch die Köpfe, noch die Hosen. Vor allen Dingen aber keinen Herrn, der sie beschützte und für sie sorgte: das wussten sie am allerhöchsten zu schätzen. (Hoffmann 1905: 181) ${ }^{19}$

Dieser gemeinschaftlich basisdemokratische, gesellschaftlich anarchistische und utopisch freiheitliche Gedanke offenbart, dass die Novelle trotz historischer Anspielungen und in der Vergangenheit existierender Gestalten, die erwähnt sind, doch keinen Bezug zur Realität hat. Ihre Aufgabe ist es, Zuversicht und Wunschvorstellungen den Pommern zu liefern, die in entlegener Provinz lebten und eine vitale Kraft aus eigenem Selbstbewusstsein kaum schöpfen konnten. Deswegen wird das Dorf „Sand“ genannt - mit der Konnotation des Unsteten, Flüchtigen, Zerstreuten. Die Ortschaft ist auf keiner Karte markiert, in keinem Amt nachweislich registriert (vgl. Hoffmann 1905: 180 f.). Dieses „herrenlos Gut“ (Hoffmann 1905: 179) zeichnet sich lediglich dadurch aus, welche natürlichen Begebenheiten es dort gibt und von wem es bewohnt ist. Die einzige vollendete Endgestalt, die

${ }^{19}$ Um des Wahrheitsgehalts der Novelle willen soll ergänzt werden, dass die neue Herrscherin Kathinka nach der Verwüstung des Dorfes zuließ, dass die Fischer etwas weiter nach Westen zogen und dort Bäume schlagen durften. Es gab also doch eine Machtstruktur, jedoch war die Herrscherin eine gutgesinnte Person. 
die Freiheit annehmen kann, ist der Charakter des Menschen: Nach einer langen Passage zum Wesen der Freiheit, fällt wohl die wichtigste identitätsstiftende Feststellung: „Lieber klein sein und seine Art behalten, als groß werden und sie verlieren“ (Hoffmann 1905: 239).

IV.

Etwas früher, gegen Mitte des 19. Jahrhunderts, verwies auch Franz Kugler, ein ausgezeichneter Kunsthistoriker Pommerns, auf die Enge der pommerschen Gedankenwelt, wobei er allerdings auch mehrere Merkmale des „poetischen Elements“ im Pommerntum unterstrich, die er bei geschichtlichen Ereignissen bestätigt zu finden glaubte:

Es mag sein, daß die Natur des pommerschen Volkes minder geschmeidig organisirt ist, als die mancher anderen Völker; daraus folgt aber gewiß nicht, daß es auch müsse arm gewesen sein an Sinn für Schönheit und Poesie, die allein dem Leben seine edlere Gestalt geben, und daß es keine genügende Kraft besessen habe, Beides zu einer höheren Vollendung zu entwickeln. Tritt doch schon in der allgemeinen Geschichte von Pommern poetisches Element genug hervor! Jene Kaufherren, deren Flotten die nordischen Meere beherrschten; jene Städte, vor deren Mauern die verbündete Macht von Fürsten und Herren vergeblich lagerte; die humoristische Laune, mit der so oft die kleinen Abenteuer in Krieg und Frieden ausgeführt wurden; die tragischen Verwickelungen, die sich häufig genug durch den unzähmbaren Freiheitsdrang des Bürgerthums bereiteten, - dies und vieles Andre sind Erscheinungen, die sich bei keinem Volke finden, dem der höhere Gehalt des Lebens fremd geblieben ist. (Kugler 1840: VIII)

Die Wechselwirkung zwischen dem ,poetischen Element“ im Alltäglichen, der gewaltvollen Geschichte, der rauen Natur und dem ungezwungenen Humor scheint auch noch um die Jahrhundertwende ein lebendiger Prozess gewesen zu sein. Es darf kein Zufall sein, dass zwischen dem Text Kuglers und Hoffmanns die literarische Periode des poetischen Realismus liegt: Im Text Hoffmanns ist die fiktionale Glaubwürdigkeitsstrategie historischen Erzählens zu finden, der Text überzeugt nicht mehr durch seine Verifizierbarkeit, sondern durch seine Präsentation im Gegensatz zur Repräsentation der faktualen Geschichtsschreibung (vgl. Dam 2016: 71 ff.). Sie liefert die Basis für die poetischen Qualitäten des Textes; diese werden gestärkt durch die Einbindung in die Geographie sowie überzeugend gezeichnete Figurentypen und die humoristische Prägung der Darstellungsweise, die jedoch nicht ins Banale abrutscht. ,[D]as vollkommen Antisentimentale“ als Eigenschaft des Hoffmannschen Humors unterstreicht Rudolf Hoffmann (Hoffmann 1926: 94); der in mehreren pommerschen Texten vorkommende bitterironische Humor gehört hier zum immanenten Element seines Glaubwürdigkeitssiegels. ${ }^{20}$

${ }^{20}$ Bereits Anfang des 20. Jahrhunderts betonte Martin Reepel verallgemeinernd, dass der Pommer ,überall die humorvolle Situation [sieht]“ (Reepel 2001: 75; Unterstreichung original); dass dieser Humor nicht banal war, bestätigte Heinz-Jürgen Zierke: „,[U]nser Humor hat immer etwas Nachdenkliches“" (Zierke 1999: 7). 
Die vielen Schichten der Novelle Hoffmanns - der historische Hintergrund und die fiktionale Ebene, der geographisch-natürliche Zusammenhang und der menschliche Kontext: Die Beschreibung der Pommern als konkrete Menschen mit ihren Charakterzügen sowie die gar märchenhafte Idealisierung der pommerschen Bürger, und nicht zuletzt die Ebene des globalen pommerschen Wesens in der Relation zum Staatswesen als auch der ethnisch-kulturellen Wahrnehmung des Selbst machen den Teufel vom Sande zu einer über das regionale hinausweisenden Novelle zur Selbstbehauptung der besonderen Eigenart innerhalb eines allgemeinen Ganzen (des Staates). Die Vielschichtigkeit der Historie und die Komplexität der menschlichen Haltungen liefern einen Beitrag zur Regional- bzw. Globalkultur, bzw. zu deren Verhältnis.

Zwar lassen sich ähnliche Aspekte bei anderen Autoren finden. Regina Hartmann stellt in ihrer Forschung zu den historischen Romanen Johann Ernst Bennos eines pommerschen Schriftstellers aus der ersten Hälfte des 19. Jahrhunderts fest, dass die Einigung Pommerns ,endlich - nun unter preußischer Ägide - zu einem dauerhaften Erfolg geführt" (Hartmann 2001: 93) habe. Auf der anderen Seite wurde jedoch der preußische Staat von Benno nicht etwa als der erwünschte Träger des nationalen Gedankens aufgefasst, der den regionalen Aspekt permanent ersetzen soll: „Wenn das Votum auch für die preußische Gegenwart ausfällt, so nicht im Sinne einer völligen Assimilation, sondern eher in dem der Wahrung der eigenen Identität" (Hartmann 2001: 94). Dass in diesem Prozess an der Schwelle des 20. Jahrhunderts der Erinnerungskultur eine besondere Rolle zuteilwurde, unterstreicht Józef Borzyszkowski: Die durch die Literatur und Bildung geförderte regionale Thematik resultierte nach ihm mit einem hohen Niveau der Volkskultur, einer besonderen Mentalität und einem „historischen Bewusstsein“ bei den Pommern (Borzyszkowski, 2002: 262, Übers. BW). Genauso - als Identitätsträger - fungiert die Geschichte bei Hoffmann: Sein narrativer Bezug auf die Vergangenheit ist dem Ziel untergeordnet, die Konstruktion des Selbst zu fördern. So gesehen übernimmt die Historie auch bei ihm - wie bei Benno - die Rolle einer ,»kontrastive[n] Spiegelgeschichte« zur preußischen Gegenwart" (Hartmann 2010: 242). Dass die preußische Identität nicht etwa als Ersatz der bisherigen angesehen werden sollte, sondern als ihre moderne Bereicherung, bezeugt die Gestalt Barnims von Kieköwer. Er füllt diesen historisch angelegten ideologischen Rahmen nahezu vollständig: Er verkörpert die musterhafte Haltung gegenüber der Wahrung alter Traditionen ${ }^{21}$ wie auch der neuen (Selbst-)Wahrnehmung. Er repräsentiert hier womöglich alle historisch wesentlichen Stände: Er war einfacher Knecht, aber auch Priester, Herr und sogar ,quasi-Herzog“. Sein Werdegang dürfte somit als eine Allegorie begriffen werden: eine Allegorie neuer Möglichkeiten und Denkhorizonte bei gleichzeitiger „frommer“ Wahrung alter Werte. Der auf

${ }^{21}$ Darauf kann auch sein Name hindeuten - Kieköwer ist ein alter pommerscher adeliger Name. 
dem Kollektivgedächtnis basierende Gedankengang Hoffmanns bleibt identitätskonkret - d.h. auf die Gruppe der ihm zeitgenössischen Pommern bezogen -, ist jedoch nicht rückwärtsgewandt. Hier kommt der utopische Ansatz des Werkes hinzu: Es darf als ein politisch-utopischer Gesellschaftstext gelesen werden, mit einem visionären Plädoyer für die Aufhebung der Ständegesellschaft. So - in einer Gemeinschaft mit einer neuen sozialen Ordnung - würde die regionale Identität zukunftsweisend verhandelt.

Eine so konstruierte identitätsstiftende Geschichte führt mehrere Profite als lokaler Kulturgutträger herbei. Die Menschen in der Region bekommen feste Orientierungspunkte, wie diese Fritz Martini in der deutschen Literatur dieser Zeit und dieses Genres findet: „Die Geschichte lehrte, einen natürlichen, kausalen und geistigen Zusammenhang des Daseins an allen seinen Stufen und Phasen zu erkennen“ (Martini 1964: 444). Die Region-Bewohner können dadurch auf der einen Seite ihren lokalen Stolz bestätigt finden, auf der anderen wird Kulturgut produziert, das die Region in kulturgeschichtlicher Hinsicht stärkt und seine Wirkung nach außen hin bekräftigt, auch wenn diese Wirkung nur innen eine Rolle spielen soll. In einer langfristigen Perspektive dürfte ein solcher Text die Region attraktiver erscheinen lassen: als ein Gebiet mit ungewöhnlichen, unverkennbaren Alleinstellungsmerkmalen. Dies wiederum bedeutet längerfristig die von außen wirkende Bestätigung des lokalen Stolzes - die nach innen hin arbeitet.

Was Hoffmann nicht vorhersehen konnte: Im heutigen Hinterpommern leben heutzutage keine Nachfahren der Menschen mehr, für die er schrieb; die Polen, auch gebürtige Westpommern, ${ }^{22}$ lesen Hoffmann nicht. ${ }^{23}$ Was aber bleibt, ist das Potential des Teufels vom Sande, auf den bis heute lebendigen Identitätsdiskurs im gegenwärtigen Hinterpommern einzuwirken.

\section{Literatur}

Arminius, Wilhelm (1909): Hans Hoffmann. Leipzig.

Bausinger, Herrmann (1990): Heimat in einer offenen Gesellschaft. Begriffsgeschichte als Problemgeschichte. In: Cremer, Will / Klein, Ansgar (Gesamtkonzeption), Bundeszentrale für politische Bildung (Hrsg.): Heimat. Analysen, Themen, Perspektiven, Schriftenreihe Bd. 294/II. Bonn. S. 76-90.

Beyerlein, Franz Adam (1906): Ein Winterlager. Berlin.

Borgmeier, Raimund / Reitz, Bernhard (Hrsg.) (1984): Der historische Roman I: 19. Jahrhundert. Heidelberg.

${ }^{22}$ Das historische Hinterpommern deckt sich fast genau mit der heutigen Wojewodschaft Westpommern (Województwo Zachodniopomorskie).

${ }^{23}$ Kein Text Hoffmanns liegt in Polnisch vor. Die Veröffentlichung einer ersten Novelle in polnischer Fassung ist für 2019 geplant. 
Borzyszkowski, Józef (2002): Kultura materialna i duchowa ludności Pomorza $w$ drugiej połowie XIX i na poczatku XX wieku. In: ders. (Hrsg.): Historia Pomorza, tom IV (1850-1918), część II: Polityka i kultura. Toruń. S. 228-270.

Buchholz, Werner et al. (1996): Historischer und geographischer Atlas von Mecklenburg und Pommern, Bd. 2, Mecklenburg und Pommern. Das Land im Rückblick. Schwerin.

Dam, Beatrix van (2016): Geschichte erzählen. Repräsentation von Vergangenheit in deutschen und niederländischen Texten der Gegenwart. Berlin.

Engel, Eduard (1908): Geschichte der Deutschen Literatur des Neunzehnten Jahrhunderts und der Gegenwart. Mit 76 Bildnissen und 20 Handschriften. Wien/Leipzig.

Erll, Astrid (2005): Kollektives Gedächtnis und Erinnerungskulturen. Eine Einführung. Stuttgart.

Fontane, Theodor (2010): Effi Briest. Husum.

Francisci, Erasmus (1663): Die lustige Schaubühne allerhand Curiositäten in einer Sprachhaltung einiger guter Freunde vorgestellet. Nürnberg, erweitert in 3 Bänden 1669-1673.

Gollnow, Ernst (1893): Ein Kreuzzug an der Ostsee. Historische Erzählung aus dem XII. Jahrhundert. Stettin.

Gollnow, Ernst (1901): Das Geheimnis der Finsternis. Ein historischer Roman aus dem XII. Jahrhundert. Stettin.

Hartmann, Horst (2010): Geschichte der Regionalliteratur Pommerns. Von den Anfängen bis zum Beginn des 20. Jahrhunderts. Aachen.

Hartmann, Regina (2001): „Die Geschichte ist die größte Dichtung“ - Johann Ernst Bennos historische Romane über Pommern. In: Baltische Studien. Pommersche Jahrbücher für Landesgeschichte Jg. 133 N. F. 87. S. 80-94.

Hartmann, Regina (2010): Geschichtskonstruktionen im kulturellen Gedächtnis: Pommern unter dem pommerschen Greif und dem preußischen Adler. In: Colloquia Germanica Stetinensia 18. S. $231-245$.

Hoffmann, Hans (1905): Der Teufel vom Sande. In: ders.: Geschichten aus Hinterpommern. Berlin. S. $145-285$.

Hoffmann, Hans (2014): Iwan der Schreckliche und sein Hund. Dresden.

Hoffmann, Hans (2013): Ruhm: Novellen. Bremen.

Rudolf Hoffmann (1926): Hans Hoffmann. Biographische Studie. In: Unser Pommerland: Monatsschrift für das Kulturleben der Heimat 11. S. 94-97.

Jensen, Wilhelm (1885): Aus den Tagen der Hansa. 3 Bde. Freiburg i. B.

Jensen, Wilhelm (1901): Ein Vorwort. In: ders.: Aus schwerer Vergangenheit. Bd. 1. Leipzig. S. VIIVIII.

Kruse, Heinrich (1881): Witzlaw von Rügen, Trauerspiel in fünf Aufzügen. Leipzig.

Kugler, Franz (1840): Pommersche Kunstgeschichte. Nach den erhaltenen Monumenten dargestellt. In: Baltische Studien. Pommersche Jahrbücher für Landesgeschichte Jg. 8, Heft 1 (ganzes Heft).

Kühlmann, Wilhelm (Hrsg.) / Killy,Walther (Begr.) (2009): Killy Literaturlexikon. Autoren und Werke des deutschsprachigen Kulturraumes. 2., vollst. überarb. Aufl. Bd. 5. Berlin (12 Bde., Berlin 2008-2012).

Ladendorf, Otto (1908): Hans Hoffmann. Sein Lebensgang und seine Werke. Berlin.

Limlei, Michael (1988): Geschichte als Ort der Bewährung. Menschenbild und Gesellschaftsverständnis in den deutschen historischen Romanen 1820-1890. Frankfurt am Main.

Martini, Fritz (1964): Deutsche Literatur im bürgerlichen Realismus 1848-1898. Stuttgart.

Meyers Großes Konversations-Lexikon. Bd. 9. Leipzig 1907.

Mumbauer, Johannes (2015): Die deutsche Dichtung der neuesten Zeit. Paderborn. Nachdruck des Originals von 1936.

Piskorski, Jan M. (Hrsg.) (1999): Pommern im Wandel der Zeiten. Szczecin. Übers. Andreas Warnecke.

Germanica Wratislaviensia 143, 2018

(C) for this edition by CNS 
Raeck, Fritz (1969): Pommersche Literatur. Proben und Daten. Nach dem Tode von Fritz Raeck zum Druck bereitet durch Rüdiger Bliß. Hamburg.

Reepel, Martin (2001): Vom Wesen des pommerschen Humors. In: Klaus Granzow: Typisch Pommern. Würzburg. S. 75-78.

Rosenfeld, Gavriel D. (2009): A Looming Crash or a Soft Landing? Forecasting the Future of the Memory „Industry“. In: The Journal of Modern History 81. S. 122-158.

Schöttgen, Christian (Hrsg.) (1721): Altes und Neues Pommerland. Oder Gesammelte Nachrichten Von verschiedenen Zur Pommerischen Historie gehörigen Stücken, Woraus die bißherigen Pommerischen Historien-Schreiber ergäntzet, verbessert und viel unbekannte Historische Wahrheiten ans Licht gebrachts werden, Aus geschriebenen und gedruckten Uhrkunden herausgegeben von Christian Schöttgen. Stettin.

Seger, Linda (1992): The Art of Adaptation. Turning Fact and Fiction into Film. New York.

[o. Autorenangabe (o. J.):] Stettin und Umgebung. Mit Illustrationen von Alex Matthaey und Ernst Schlemo (=Städtebilder und Landschaften aus aller Welt No. 119-119a). Zürich.

Tylor, Edward B. (1873): Die Anfänge der Cultur. Untersuchungen über die Entwicklung der Mythologie, Philosophie, Religion, Kunst und Sitte. Leipzig. Englischsprachige Erstausgabe: Primitive Culture, 1871.

Werder, Hans (1901): Der Pommernherzog. Roman aus alter Zeit. Berlin.

Wisniewski, Roswitha (2013): Geschichte der deutschen Literatur Pommerns. Vom Mittelalter bis zum Beginn des 21. Jahrhunderts. Berlin.

Heinz-Jürgen Zierke (1999): Pommern grient. Rostock.

Germanica Wratislaviensia 143, 2018

(C) for this edition by CNS 